\title{
PREDICTORS OF SHORT- AND LONG-TERM SICKNESS ABSENCE IN FEMALE POST OFFICE WORKERS IN POLAND
}

\section{ZUZANNA SZUBERT $^{1}$, TERESA MAKOWIEC-DĄBROWSKA ${ }^{2}$, DOROTA MERECZ ${ }^{3}$, and WOJCIECH SOBALA ${ }^{1}$}

Nofer Institute of Occupational Medicine, Łódź, Poland

${ }^{1}$ Department of Environmental Epidemiology

${ }^{2}$ Department of Work Physiology and Ergonomics

${ }^{3}$ Department of Work Psychology

\begin{abstract}
Background: The aim of this study was to highlight major predictors of the frequency of sickness absence in a group of workers directly involved in customer service. Material and Methods: The study was carried out on a random sample of 229 women employed as assistants and clerks in post offices. The survey was based on the Subjective Work, Health Status and Life Style Characteristics Questionnaire, and sickness absence data for the years 2004-2006. Results: The negative binominal regression model of sickness absence risk revealed the following significant predictors of short-term absence spells (1-29 days): 1) marital status, sickness absence risk for single women was (rate ratio (RR)) $=1.56$ (95\% confidence interval (CI): 1.01-2.39) vs. married women; 2 ) post offices employing 7 workers had a rate ratio of sickness absence of 1.6 (95\% CI: 1.04-2.42); 13-25 workers - RR = 2.03 (95\% CI: $1.41-2.93) ;>25$ workers - RR = 1.82 (95\% CI: 1.15-2.88) compared with an average number of 8-12 workers; 3 ) shift work, RR $=1.57$ (95\% CI: 1.14-2.14); 4) breaks from work the risk of absence in the case of any breaks amounted to RR $=1.5$ (95\% CI: 1.07-2.07) in comparison with the statutory breaks; 5) self-rated health reported as moderate relative to good health, $\mathrm{RR}=1.71$ (95\% CI: 1.26-2.32); and 6) occurrence of respiratory diseases resulted in the risk of $\mathrm{RR}=1.51$ (95\% CI: 1.08-2.08). The Poisson regression model of long-term sickness absence spells ( $\geq 30$ days) revealed the following significant predictors: 1 ) number of clients per shift: $51-100$ clients, $\mathrm{RR}=3.62$ (95\% CI: 1.07-22.6) compared with a lower number of clients; 2 ) self-rated health, assessed as moderate, $\mathrm{RR}=1.97$ (95\% CI: 1.06-3.78) and 3) household chores performed for at least $4 \mathrm{~h}$ a day, RR $=0.4$ (95\% CI: $0.18-0.79$ ). Conclusions: Association between sickness absence and workload as well as work organization indicates directions of corrective actions, which could reduce the scale of the problem.
\end{abstract}

Key words:

Sick leave, Working conditions, Post office workers, Psychosocial workload, Risk factors, Health status

\section{INTRODUCTION}

Social and economic transformations as well as labor market situation exert certain effects on the indicators that reflect the health status of occupationally active people, as well as on their sickness absence, namely a temporary inability to work caused by a disease or an accident. Sickness absence is also influenced by non-health determinants, such as: demographic characteristics of the population, working conditions, psychosocial, economic and cultural factors, economic status, systems of health insurance

This work was performed under the research item IMP 10.3 "Physical and psychosocial determinants of sickness absence in workers employed in the customer service sector." Project manager: Zuzanna Szubert, Ph.D.

Received: April 10, 2013. Accepted: July 3, 2015.

Corresponding author: Z. Szubert, Nofer Institute of Occupational Medicine, Department of Environmental Epidemiology, św. Teresy 8, 91-348 Łódź, Poland (e-mail: zuzanna@imp.lodz.pl). 
and social security [1-4]. During the period of system transformation in Poland, especially changes in the area of employment, the influence of these factors and determinants took a quite different dimension. The extent to which sickness absence reflects the actual health status of the population may be associated with the more and more frequent in recent years phenomenon - "presenteeism," i.e., taking up work despite an illness [5,6].

In the majority of economically developed countries, the data on sickness absence from work and its causes are a source of information on, and measure of, health status of different occupational groups [3,7-9]. Nevertheless, the possibility of international comparison is limited since the results of analyses of inability to work depend greatly on the medical certification legislation and health insurance systems in individual countries [10-12].

During a period of restructuring (including job losses) in numerous economic organizations and institutions certain disturbances emerge in the labor environment (negative attitudes towards job, conflicts, unsettled ties) that, given the situation on the labor market (unemployment, high competitiveness), increase levels of occupational stress. These determinants influence, both directly and indirectly, the incidence of sickness absence through inducing changes in workers' health status [4,13-16].

Owing to structural changes in the Polish economy, the number of workers employed in the service sector has been growing. Occupational groups involved in direct customer service (e.g., banks, post offices) have not as yet been the subject of studies focused on the analysis of sickness absence determinants in Poland.

The analysis of the frequency and severity of absence spells and the effect of variables that characterize physical and psychosocial working conditions may play a significant role in introducing actions aimed at diminishing negative consequences of sickness absence, such as disorganization of work, quality of provided services and enhanced labor costs [17-19]. Moreover, one should take into account the adverse consequences resulting from health deterioration associated, among others, with the nature of the job. However, one should remember that sickness absence is a complex issue determined by the health of an employee, as well as by psychosocial and economic factors affecting approach towards health, work and real working conditions $[1,2,20]$.

The aim of this study was to highlight major predictors, especially those characteristic of physical and psychosocial working conditions, which significantly influence the frequency of sickness absence in a group of workers directly involved in customer service.

\section{MATERIAL AND METHODS}

\section{Study group}

The study group comprised female workers employed directly in customer service in post offices of a large urban agglomeration. The study was performed on a random sample of 229 women employed as postal assistants and clerks to assure the homogeneity of the study group in terms of the nature of the job (90\% of postal service employees are women). The study had a stratified cluster randomization design, which means that a post office was adopted as a randomization unit. The diversified nature of work, depending on the size of a given setting - the larger the number of employees in a post office, the wider their tasks (servicing of delivery workers, receipt of packages) and a shift work system justified selection of this scheme. Sixty-seven post offices were divided into 4 groups (with the following number of the employed women in each group: 2-7, 8-12, 13-25, $\geq 26$ ). The number of 29 selected offices in the groups was: $13,10,5,1$, respectively. From each randomly selected post office, all women working on work posts mentioned before were included in the study. Owing to the fact that the studied group embraced over 550 women, to ensure the representation of post offices in each sample, post offices of different sizes made up about $1 / 3$ of the sample. 
In practice, performing interviews, as originally proposed, with the whole group of 272 workers employed in the randomized post offices proved impossible. Of this number 28 women refused to participate in the study, 11 were absent for a long time because of maternity and childcare leave, retirement or transfer to other post offices. Therefore, empirical data covering both sickness absence and questionnaire-based interview were collected from 233 women employed directly on customer service posts. Pregnant women were excluded from the analysis since their longterm absence from work was frequently related to antenatal care. Finally, the sample covered 229 women.

\section{Variables}

The analysis included the following groups of variables:

- demographic characteristics: age, marital status, education;

- characteristics of physical working conditions: working time, shifts, breaks at work, job description, workload (defined by the work energy expenditure per shift - kcal/shift);

- subjective indicators of workload: fatigue after work, tiring factors at work;

- psychosocial characteristics of work;

- self-reported health status: list of illnesses and complaints, including the year of their onset (diseases occurring prior to the sickness absence were classified for the analysis);

- lifestyle characteristics, such as: cigarette smoking, alcohol consumption, overload with household chores, forms of leisure time activities;

- stressful life events, such as: a divorce, death of a family member or a close friend, the respondent's own disease, marriage, change in economic situation and so forth (in total 27 events) along with a 4-rating scale scores concerning negative emotions induced by those events;

- spells of inability to work because of a disease or an accident in the years 2004-2006.

\section{Sources of information}

The Individual Sickness Absence Card (ISAC), developed for the purpose of this study, constituted the basis for collecting data on sickness absence. Medical certificates of employees' temporary inability to work in the period between January 1, 2004 and December 31, 2006 collected by an employer constituted the source of information. All cases of inability to work starting in that period, regardless of the date of their termination, were included in ISAC.

The questionnaire study was performed in the first half of 2007. The Health Status and Lifestyle Questionnaire was a source of information about physical characteristics of work, health status and workers' lifestyle characteristics. The Subjective Work Characteristics QuestionnaireRevised (SWCQ-R), an updated version of the original SWCQ developed by Dudek et al. in 1999 [21], was used to assess the level of occupational stress. The SWCQ and SWCQ-R were designed to measure occupational stress in workers. A revised version of SWCQ was prepared to update the reference standards for the questionnaire results. Factor analysis was performed on the data coming from over 3000 employees.

Version of the questionnaire used in this study comprised 53 items with a Likert-type 5-point response scale from 1 ("this characteristics is not relevant to my job") to 5 ("it constantly irritates me at work and makes me nervous even at home"). In its new version, the number of items was reduced to 50 and the number of factors to 5 . This was justified by the results of the confirmatory factor analysis. Thus, the 5 subscales of the questionnaire distinguished were, as follows: 1) tension and pressure, 2) hazards, 3) psychic workload, 4) lack of control over work, 5) unpleasant working conditions. To estimate sickness absence risk, the results obtained by the respondents in these subscales were divided into 3 categories: low, average and high.

Polish adaptation of the Holmes-Rahe Social Readjustment Rating Scale (1967) was used to assess life stress 
burden. The scale originally measured the amount of change, using Life Change Units, a person experienced and adjusted to in the previous 12 months. It was designed to predict the likelihood of a disease and illness following exposure to stressful life events [22]. In the Polish adaptation the level of stress related to a particular event is also assessed.

The level of sickness absence in a 3-year period was defined by means of 2 parameters (calculated as an average annual rate):

- the rate of absence spells, i.e., the number of sickness absence spells per 100 person-days;

- the rate of sickness absence calculated as a ratio of the number of sick leave days to the number of persondays (expressed as a percentage).

\section{Statistical analysis}

Statistical analysis was performed by the use of a twosided tests; a value of $p=0.05$ was considered as statistically significant. Confidence intervals for absence rates (the number of days and the number of periods) were calculated using the nonparametric Bootstrap method [23]. Ten thousand new data sets were created by sampling with replacement from the original data set. For each data set, relevant rates were calculated and its $95 \%$ confidence interval as percentiles of the relevant rate distribution.

To analyze the absence spell rate it was assumed that the factors shaping sickness absence may be diverse, depending on the length of absence, i.e., severity of illness. Separate analyses were performed for periods of 1-29 days, 30 days and more. Distribution of periods of absence due to a disease or an accident is presented in Table 1.

Impact of the variables on the rate of absence spells was analyzed using a negative binominal regression model (for short-term spells) and the Poisson regression model (for long-term spells). We used the negative binomial regression to model overdispersion for short-term spells. For
Table 1. Sickness absence spells among the female post office clerks in 2004-2006

\begin{tabular}{lcc}
\hline & $\begin{array}{c}\text { Absence } \\
\text { [days] }\end{array}$ & $\begin{array}{c}\text { Absence spells } \\
{[\mathrm{n}(\%)]}\end{array}$ \\
\hline $1-3$ & & $8(3.0)$ \\
$4-9$ & $102(38.1)$ \\
$10-14$ & $46(17.2)$ \\
$15-29$ & $58(21.6)$ \\
$30-89$ & $47(17.5)$ \\
$\geq 90$ & $7(2.6)$ \\
Total & $268(100.0)$ \\
\hline
\end{tabular}

long-term spells we did observe overdispersion for the intercept-only-model. In the models applied, the number of absence spells in the observation period was a dependent variable and the logarithm of the number of observation person-days was introduced as an offset. In the calculation of person-days, the periods in which a given person was absent from work because of a disease, accident, taking care of a family member or maternity leave were excluded. In the first phase of the study a univariate regression analysis was performed. This was followed by a multivariable analysis of variables significant at a level of $p=0.2$. In the multivariable analysis the variables were eliminated from the model using a stepwise method, applying the Akaike Information Criterion (AIC) as an exclusion criterion [24]. We used AIC criterion to select a model with a good predictive performance. All the statistical analyses were performed employing R-statistic package, version 2.15.2 [25]. The Poisson regression model was fitted using glm function from stats library, and the negative binominal regression model using glm.nb function from MASS library, version 7.3-23 [26].

\section{RESULTS}

\section{Sickness absence rates}

The analysis showed that of the 229 women, 89 (39\%) had no period of registered absence because of a disease or 
an accident in the years 2004-2006. In the remaining group of women, 268 absence spells of 2-229 days were registered in that period. Eighty percent were absence spells of 2-29 days and were responsible for 2456 (43\%) days of sickness absence, and 54 absence spells of 30 days and more caused 3306 (57\%) days of absence at work. On average, over 25 days of absence per woman employed as a postal assistant or clerk were registered during the period under the study.

The average annual rate of sickness absence days (expressed as a percentage of non-working time) was 8.9, while the rate of absence spells was 41.4 (Table 2). The data presented in this table indicate the diversification of the absence extent (expressed as a period of absence) and the frequency of absence (expressed as absence days), depending on the year of the study, age, duration of employment and earlier occurrence of major categories of illnesses. Starting from the age of 30 , the absence rate, expressed in days, increased along with age, while the highest frequency of absence was observed in the youngest and the oldest age groups. In terms of employment duration, sickness absence showed considerable fluctuations both in the rate of days and in the rate of periods of absence. The highest rates were noted in the women with the shortest duration of employment. Occurrence of any kind of illness was coupled with a higher level of absence; the highest rates were noted due to accident-related injuries (Table 2).

Table 2. Sickness absence among the female post office clerks in 2004-2006

\begin{tabular}{|c|c|c|c|c|c|}
\hline \multirow{3}{*}{ Variable } & \multirow{3}{*}{$\begin{array}{c}\text { Respondents } \\
(\mathrm{N}=229) \\
{[\mathrm{n}]}\end{array}$} & \multicolumn{4}{|c|}{ Absence } \\
\hline & & \multicolumn{2}{|c|}{ spells } & \multicolumn{2}{|c|}{ days } \\
\hline & & rate $^{\mathrm{a}}$ & $95 \% \mathrm{CI}$ & rate $^{\mathrm{b}}$ & $95 \% \mathrm{CI}$ \\
\hline \multicolumn{6}{|l|}{ Year } \\
\hline 2004 & 213 & 40.5 & $31.8-49.9$ & 9.5 & 6.4-13.1 \\
\hline 2005 & 219 & 42.1 & $33.8-50.8$ & 6.8 & $4.9-8.9$ \\
\hline 2006 & 229 & 41.6 & $32.8-50.6$ & 10.4 & $7.3-13.9$ \\
\hline \multicolumn{6}{|c|}{ Age during the interview [years] } \\
\hline$\leq 29$ & 22 & 55.1 & $26.2-90.7$ & 8.1 & $3.2-13.7$ \\
\hline $30-39$ & 85 & 37.0 & $28.3-46.3$ & 6.9 & $4.5-9.9$ \\
\hline $40-49$ & 88 & 39.3 & $30.9-48.5$ & 9.8 & $7.1-12.8$ \\
\hline$\geq 50$ & 34 & 51.0 & $34.7-68.1$ & 11.9 & $6.8-17.9$ \\
\hline \multicolumn{6}{|c|}{ Duration of employment [years] } \\
\hline $1-5$ & 63 & 46.4 & $33.4-61.3$ & 11.9 & 7.1-17.4 \\
\hline $6-10$ & 38 & 37.7 & $25.0-51.7$ & 6.3 & $3.7-9.7$ \\
\hline $11-20$ & 84 & 41.7 & $32.0-52.3$ & 8.6 & $6.2-11.4$ \\
\hline$\geq 21$ & 44 & 38.6 & $27.6-50.0$ & 8.3 & $5.3-11.6$ \\
\hline \multicolumn{6}{|l|}{$\begin{array}{l}\text { Occurrence of the diseases } \\
\text { accident-related injury }\end{array}$} \\
\hline yes & 8 & 60.8 & $27.6-91.8$ & 16.8 & $5.8-31.5$ \\
\hline no & 221 & 40.7 & $34.6-46.9$ & 8.6 & $6.9-10.5$ \\
\hline \multicolumn{6}{|c|}{ musculoskeletal system diseases } \\
\hline yes & 83 & 43.0 & $32.8-54.0$ & 9.7 & $6.8-12.8$ \\
\hline no & 146 & 40.5 & $33.4-48.1$ & 8.5 & $6.4-10.8$ \\
\hline
\end{tabular}


Table 2. Sickness absence among the female post office clerks in 2004-2006 - cont.

\begin{tabular}{|c|c|c|c|c|c|}
\hline \multirow{3}{*}{ Variable } & \multirow{3}{*}{$\begin{array}{c}\text { Respondents } \\
(\mathrm{N}=229) \\
{[\mathrm{n}]}\end{array}$} & \multicolumn{4}{|c|}{ Absence } \\
\hline & & \multicolumn{2}{|c|}{ spells } & \multicolumn{2}{|c|}{ days } \\
\hline & & rate $^{a}$ & $95 \% \mathrm{CI}$ & rate $^{b}$ & $95 \% \mathrm{CI}$ \\
\hline \multicolumn{6}{|c|}{$\begin{array}{l}\text { Occurrence of the diseases - cont. } \\
\text { circulatory system diseases }\end{array}$} \\
\hline yes & 44 & 51.2 & $34.2-69.3$ & 10.6 & $6.7-15.1$ \\
\hline no & 185 & 39.1 & $33.0-45.6$ & 8.5 & $6.7-10.6$ \\
\hline \multicolumn{6}{|c|}{ respiratory system diseases } \\
\hline yes & 42 & 60.8 & $42.1-80.5$ & 12.8 & $8.3-17.8$ \\
\hline no & 187 & 37.2 & $31.4-43.3$ & 8.0 & $6.3-10.1$ \\
\hline \multicolumn{6}{|l|}{ mental disorders } \\
\hline yes & 19 & 47.4 & $28.2-68.4$ & 11.5 & $4.3-20.8$ \\
\hline no & 210 & 40.9 & $34.7-47.4$ & 8.7 & $7.0-10.5$ \\
\hline \multicolumn{6}{|l|}{ nervous system diseases } \\
\hline yes & 62 & 40.6 & $29.1-53.2$ & 9.6 & $6.0-13.7$ \\
\hline no & 167 & 41.7 & $35.1-49.0$ & 8.6 & $6.8-10.8$ \\
\hline \multicolumn{6}{|l|}{ digestive system diseases } \\
\hline yes & 40 & 53.4 & $39.4-68.6$ & 11.9 & $8.2-15.9$ \\
\hline no & 189 & 38.8 & $32.4-45.6$ & 8.3 & $6.4-10.4$ \\
\hline \multicolumn{6}{|c|}{ genitourinary system diseases } \\
\hline yes & 27 & 44.5 & $28.9-61.3$ & 10.4 & $5.4-16.2$ \\
\hline no & 202 & 41.0 & $34.6-47.8$ & 8.7 & $6.9-10.7$ \\
\hline \multicolumn{6}{|l|}{ skin diseases } \\
\hline yes & 16 & 50.7 & $28.1-76.5$ & 15.7 & $8.2-23.8$ \\
\hline no & 213 & 40.7 & $34.7-46.9$ & 8.4 & $6.7-10.2$ \\
\hline \multicolumn{6}{|l|}{ neoplasms } \\
\hline yes & 5 & 60.0 & $16.7-105.5$ & 11.4 & $1.0-26.5$ \\
\hline no & 224 & 41.0 & $35.0-47.2$ & 8.8 & $7.2-10.7$ \\
\hline \multicolumn{6}{|l|}{ endocrine disorders } \\
\hline yes & 17 & 55.1 & $37.5-74.2$ & 14.6 & $6.2-24.9$ \\
\hline no & 212 & 40.3 & $34.2-46.7$ & 8.4 & $6.8-10.3$ \\
\hline \multicolumn{6}{|c|}{ diseases of blood and blood-forming } \\
\hline yes & 8 & 41.6 & $16.7-75.0$ & 11.6 & $3.6-21.7$ \\
\hline no & 221 & 41.4 & $35.4-47.8$ & 8.8 & $7.1-10.7$ \\
\hline Total & 229 & 41.4 & $35.5-47.6$ & 8.9 & $7.2-10.8$ \\
\hline
\end{tabular}

CI - confidence interval.

${ }^{\text {a }}$ Sickness absence spells rate $=$ the number of spells per 100 person-days.

${ }^{\mathrm{b}}$ Sickness absence rate $=$ the number of sick leave days/number of person-days $\times 100$.

\section{Univariate analysis}

The findings of the absence risk analysis by variable is presented in Table 3. Of the demographic variables, such as education, marital status, age or duration of employment, the risk of absence of 1-29 days was significantly increased (by 76\%) in the unmarried women compared 
Table 3. Distribution of persons and sickness absence risk - univariate analyses

\begin{tabular}{|c|c|c|c|c|c|c|c|}
\hline \multirow[b]{3}{*}{ Variable } & \multirow{3}{*}{$\begin{array}{c}\text { Respondents } \\
(\mathrm{N}=229) \\
{[\mathrm{n}]}\end{array}$} & \multicolumn{6}{|c|}{ Duration of spells } \\
\hline & & \multicolumn{3}{|c|}{ 1-29 days } & \multicolumn{3}{|c|}{$\geq 30$ days } \\
\hline & & $\begin{array}{c}\text { spells } \\
(\mathrm{N}=214) \\
{[\mathrm{n}]}\end{array}$ & RR & $95 \% \mathrm{CI}$ & $\begin{array}{c}\text { spells } \\
(\mathrm{N}=54) \\
{[\mathrm{n}]}\end{array}$ & $\mathrm{RR}$ & $95 \% \mathrm{CI}$ \\
\hline \multirow{2}{*}{\multicolumn{8}{|c|}{$\begin{array}{l}\text { Demographic characteristics } \\
\text { education }\end{array}$}} \\
\hline & & & & & & & \\
\hline primary, vocational & 14 & 13 & 1.00 & reference & 5 & 1.00 & reference \\
\hline secondary & 179 & 177 & 1.11 & $0.58-2.26$ & 43 & 0.70 & $0.31-2.03$ \\
\hline university & 36 & 24 & 0.86 & $0.39-1.94$ & 6 & 0.56 & $0.17-1.94$ \\
\hline \multicolumn{8}{|l|}{ marital status } \\
\hline married & 158 & 138 & 1.00 & reference & 39 & 1.00 & reference \\
\hline unmarried & 27 & 38 & 1.76 & $1.12-2.73^{*}$ & 7 & 1.15 & $0.47-2.41$ \\
\hline cohabiting & 10 & 14 & 1.58 & $0.78-3.09$ & 4 & 1.58 & $0.47-3.92$ \\
\hline divorced, widowed & 34 & 24 & 0.79 & $0.47-1.28$ & 4 & 0.47 & $0.14-1.16$ \\
\hline \multicolumn{8}{|l|}{ age [years] } \\
\hline$\leq 29$ & 22 & 25 & 1.00 & reference & 3 & 1.00 & reference \\
\hline $30-39$ & 85 & 75 & 0.64 & $0.37-1.12$ & 14 & 0.98 & $0.32-4.26$ \\
\hline $40-49$ & 88 & 76 & 0.61 & $0.35-1.07$ & 26 & 1.71 & $0.60-7.16$ \\
\hline$\geq 50$ & 34 & 38 & 0.82 & $0.44-1.54$ & 11 & 1.96 & $0.61-8.67$ \\
\hline \multicolumn{8}{|c|}{ duration of employment [years] } \\
\hline$\leq 5$ & 63 & 53 & 1.00 & reference & 16 & 1.00 & reference \\
\hline $6-10$ & 38 & 38 & 0.93 & $0.56-1.54$ & 5 & 0.40 & $0.13-1.03$ \\
\hline $11-20$ & 84 & 84 & 0.94 & $0.62-1.43$ & 21 & 0.77 & $0.40-1.49$ \\
\hline$\geq 21$ & 44 & 39 & 0.83 & $0.50-1.36$ & 12 & 0.84 & $0.39-1.76$ \\
\hline \multirow{2}{*}{\multicolumn{8}{|c|}{$\begin{array}{l}\text { Working conditions and workload } \\
\text { post office clerks [n] }\end{array}$}} \\
\hline & & & & & & & \\
\hline $2-7$ & 44 & 42 & 1.69 & $1.07-2.65^{*}$ & 5 & 0.55 & $0.18-1.35$ \\
\hline $8-12$ & 95 & 54 & 1.00 & reference & 20 & 1.00 & reference \\
\hline $13-25$ & 63 & 81 & 2.24 & $1.52-3.33^{*}$ & 19 & 1.43 & $0.76-2.69$ \\
\hline$\geq 26$ & 27 & 37 & 2.33 & $1.43-3.77^{*}$ & 10 & 1.70 & $0.76-3.55$ \\
\hline \multicolumn{8}{|l|}{ working hours } \\
\hline full-time employment & 51 & 66 & 1.00 & reference & 12 & 1.00 & reference \\
\hline part-time employment & 178 & 148 & 0.66 & $0.46-0.94^{*}$ & 42 & 1.03 & $0.56-2.04$ \\
\hline \multicolumn{8}{|l|}{ shift work $[\mathrm{n}]$} \\
\hline 1 & 17 & 17 & 1.24 & $0.66-2.21$ & 1 & 0.27 & $0.02-1.24$ \\
\hline 2 & 171 & 137 & 1.00 & reference & 37 & 1.00 & reference \\
\hline 3 & 41 & 60 & 1.81 & $1.24-2.63^{*}$ & 16 & 1.77 & $0.96-3.13$ \\
\hline
\end{tabular}


Table 3. Distribution of persons and sickness absence risk - univariate analyses - cont.

\begin{tabular}{|c|c|c|c|c|c|c|c|}
\hline \multirow[b]{3}{*}{ Variable } & \multirow{3}{*}{$\begin{array}{c}\text { Respondents } \\
(\mathrm{N}=229) \\
{[\mathrm{n}]}\end{array}$} & \multicolumn{6}{|c|}{ Duration of spells } \\
\hline & & \multicolumn{3}{|c|}{ 1-29 days } & \multicolumn{3}{|c|}{$\geq 30$ days } \\
\hline & & $\begin{array}{c}\text { spells } \\
(\mathrm{N}=214) \\
{[\mathrm{n}]}\end{array}$ & $\mathrm{RR}$ & $95 \% \mathrm{CI}$ & $\begin{array}{c}\text { spells } \\
(\mathrm{N}=54) \\
{[\mathrm{n}]}\end{array}$ & $\mathrm{RR}$ & $95 \% \mathrm{CI}$ \\
\hline \multicolumn{8}{|c|}{$\begin{array}{l}\text { Working conditions and workload - cont. } \\
\text { overtime work [times/month] }\end{array}$} \\
\hline no overtime work & 105 & 89 & 1.00 & reference & 26 & 1.00 & reference \\
\hline $1-5$ & 71 & 77 & 1.33 & $0.92-1.92$ & 16 & 0.95 & $0.50-1.75$ \\
\hline $6-10$ & 29 & 21 & 0.85 & $0.48-1.46$ & 5 & 0.70 & $0.24-1.67$ \\
\hline $11-25$ & 24 & 27 & 1.34 & $0.78-2.25$ & 7 & 1.18 & $0.47-2.58$ \\
\hline \multicolumn{8}{|l|}{ breaks at work } \\
\hline set in working time regulation & 148 & 136 & 1.00 & reference & 42 & 1.00 & reference \\
\hline depending on the needs & 41 & 51 & 1.39 & $0.93-2.07$ & 5 & 0.44 & $0.15-1.01$ \\
\hline no breaks & 40 & 27 & 0.80 & $0.49-1.27$ & 7 & 0.66 & $0.27-1.38$ \\
\hline \multicolumn{8}{|c|}{ time spent on the computer daily [h] } \\
\hline$\leq 4$ & 25 & 26 & 1.00 & reference & 3 & 1.00 & reference \\
\hline $4.5-6$ & 109 & 93 & 0.83 & $0.49-1.43$ & 22 & 1.71 & $0.59-7.23$ \\
\hline $6.5-8$ & 95 & 95 & 0.97 & $0.57-1.60$ & 29 & 2.57 & $0.92-10.75$ \\
\hline \multicolumn{8}{|l|}{ computer screen location } \\
\hline in front & 12 & 19 & 1.00 & reference & 2 & 1.00 & reference \\
\hline aside & 217 & 195 & 0.60 & $0.33-1.13$ & 52 & 1.53 & $0.48-9.35$ \\
\hline \multicolumn{8}{|l|}{ upper edge of the computer monitor } \\
\hline at eye level & 114 & 104 & 1.00 & reference & 30 & 1.00 & reference \\
\hline below eye level & 44 & 43 & 1.07 & $0.69-1.64$ & 10 & 0.86 & $0.40-1.70$ \\
\hline above eye level & 71 & 67 & 1.05 & $0.72-1.51$ & 14 & 0.76 & $0.39-1.41$ \\
\hline \multicolumn{8}{|l|}{$\begin{array}{l}\text { light reflection on the computer } \\
\text { monitor }\end{array}$} \\
\hline no & 102 & 99 & 1.00 & reference & 23 & 1.00 & reference \\
\hline yes & 127 & 115 & 0.95 & $0.69-1.32$ & 31 & 1.10 & $0.64-1.91$ \\
\hline \multicolumn{8}{|l|}{ wearing glasses } \\
\hline no & 115 & 108 & 1.00 & reference & 23 & 1.00 & reference \\
\hline yes & 114 & 106 & 0.93 & $0.67-1.28$ & 31 & 1.27 & $0.74-2.20$ \\
\hline \multicolumn{8}{|l|}{ keyboard position } \\
\hline on the tabletop & 162 & 156 & 1.00 & reference & 41 & 1.00 & reference \\
\hline on the pull-out extension & 67 & 58 & 0.94 & $0.65-1.34$ & 13 & 0.80 & $0.41-1.46$ \\
\hline \multicolumn{8}{|l|}{ mouse position } \\
\hline in front of the keyboard & 50 & 39 & 1.00 & reference & 9 & 1.00 & reference \\
\hline next to the keyboard & 179 & 175 & 1.18 & $0.79-1.80$ & 45 & 1.32 & $0.68-2.88$ \\
\hline
\end{tabular}


Table 3. Distribution of persons and sickness absence risk - univariate analyses - cont.

\begin{tabular}{|c|c|c|c|c|c|c|c|}
\hline \multirow[b]{3}{*}{ Variable } & \multirow{3}{*}{$\begin{array}{c}\text { Respondents } \\
(\mathrm{N}=229) \\
{[\mathrm{n}]}\end{array}$} & \multicolumn{6}{|c|}{ Duration of spells } \\
\hline & & \multicolumn{3}{|c|}{ 1-29 days } & \multicolumn{3}{|c|}{$\geq 30$ days } \\
\hline & & $\begin{array}{c}\text { spells } \\
(\mathrm{N}=214) \\
{[\mathrm{n}]} \\
\end{array}$ & $\mathrm{RR}$ & $95 \% \mathrm{CI}$ & $\begin{array}{c}\text { spells } \\
(\mathrm{N}=54) \\
{[\mathrm{n}]}\end{array}$ & $\mathrm{RR}$ & $95 \% \mathrm{CI}$ \\
\hline \multicolumn{8}{|c|}{$\begin{array}{l}\text { Working conditions and workload - cont. } \\
\text { clients [n/shift] }\end{array}$} \\
\hline$\leq 50$ & 25 & 24 & 1.00 & reference & 2 & 1.00 & reference \\
\hline $51-100$ & 95 & 89 & 0.97 & $0.57-1.71$ & 28 & 3.68 & $1.11-22.81 *$ \\
\hline $101-150$ & 67 & 62 & 0.94 & $0.53-1.69$ & 18 & 3.26 & $0.94-20.49$ \\
\hline$\geq 151$ & 37 & 33 & 0.92 & $0.49-1.75$ & 5 & 1.66 & $0.36-11.61$ \\
\hline \multicolumn{8}{|c|}{$\begin{array}{l}\text { slight bending of the trunk } \\
\text { [\% of working time] }\end{array}$} \\
\hline$<25 \%$ & 42 & 32 & 0.75 & $0.46-1.18$ & 5 & 0.37 & $0.13-0.88^{*}$ \\
\hline $25-75 \%$ & 87 & 82 & 0.95 & $0.66-1.35$ & 18 & 0.67 & $0.37-1.18$ \\
\hline$>75 \%$ & 100 & 100 & 1.00 & reference & 31 & 1.00 & reference \\
\hline \multicolumn{8}{|c|}{ extensive bending of the trunk } \\
\hline no & 178 & 174 & 1.00 & reference & 41 & 1.00 & reference \\
\hline yes & 51 & 40 & 0.83 & $0.55-1.24$ & 13 & 1.15 & $0.59-2.08$ \\
\hline \multicolumn{8}{|c|}{ twisted trunk [\% of working time] } \\
\hline no & 90 & 92 & 1.00 & reference & 25 & 1.00 & reference \\
\hline yes, $\leq 25 \%$ & 102 & 97 & 0.96 & $0.68-1.36$ & 22 & 0.80 & $0.45-1.43$ \\
\hline yes, $>25 \%$ & 37 & 25 & 0.68 & $0.40-1.12$ & 7 & 0.70 & $0.28-1.54$ \\
\hline \multicolumn{8}{|l|}{ squatting position } \\
\hline no & 197 & 183 & 1.00 & reference & 47 & 1.00 & reference \\
\hline yes & 32 & 31 & 1.10 & $0.69-1.74$ & 7 & 0.96 & $0.40-1.99$ \\
\hline \multicolumn{8}{|c|}{ physical workload rate [kcal/shift] } \\
\hline $126-250$ & 37 & 32 & 1.00 & reference & 12 & 1.00 & reference \\
\hline $251-400$ & 146 & 143 & 1.13 & $0.72-1.81$ & 35 & 0.75 & $0.40-1.51$ \\
\hline $401-1300$ & 46 & 39 & 1.00 & $0.57-1.76$ & 7 & 0.48 & $0.18-1.20$ \\
\hline \multicolumn{8}{|c|}{$\begin{array}{l}\text { Subjective indicators of workload } \\
\text { fatigue after work }\end{array}$} \\
\hline low & 45 & 44 & 1.00 & reference & 7 & 1.00 & reference \\
\hline moderate & 80 & 65 & 0.89 & $0.56-1.42$ & 16 & 1.39 & $0.59-3.61$ \\
\hline heavy & 104 & 105 & 1.11 & $0.73-1.71$ & 31 & 2.06 & $0.96-5.10$ \\
\hline \multicolumn{8}{|c|}{$\begin{array}{l}\text { level of fatigue vs. most extensive } \\
\text { fatigue [\%] }\end{array}$} \\
\hline$\leq 30$ & 66 & 68 & 1.00 & reference & 12 & 1.00 & reference \\
\hline $31-70$ & 89 & 76 & 0.88 & $0.59-1.31$ & 23 & 1.51 & $0.77-3.14$ \\
\hline $71-100$ & 74 & 70 & 0.98 & $0.65-1.47$ & 19 & 1.51 & $0.74-3.19$ \\
\hline
\end{tabular}


Table 3. Distribution of persons and sickness absence risk - univariate analyses - cont.

\begin{tabular}{|c|c|c|c|c|c|c|c|}
\hline \multirow[b]{3}{*}{ Variable } & \multirow{3}{*}{$\begin{array}{c}\text { Respondents } \\
(\mathrm{N}=229) \\
{[\mathrm{n}]}\end{array}$} & \multicolumn{6}{|c|}{ Duration of spells } \\
\hline & & \multicolumn{3}{|c|}{ 1-29 days } & \multicolumn{3}{|c|}{$\geq 30$ days } \\
\hline & & $\begin{array}{c}\text { spells } \\
(\mathrm{N}=214) \\
{[\mathrm{n}]}\end{array}$ & $\mathrm{RR}$ & $95 \% \mathrm{CI}$ & $\begin{array}{c}\text { spells } \\
(\mathrm{N}=54) \\
{[\mathrm{n}]}\end{array}$ & $\mathrm{RR}$ & $95 \% \mathrm{CI}$ \\
\hline \multicolumn{8}{|c|}{$\begin{array}{l}\text { Subjective indicators of workload - cont. } \\
\text { tiring factors at work } \\
\text { work at different times of the day }\end{array}$} \\
\hline no & 180 & 153 & 1.00 & reference & 199 & 1.00 & reference \\
\hline yes & 49 & 61 & 1.48 & $1.02-2.13^{*}$ & 15 & 1.42 & $0.76-2.51$ \\
\hline \multicolumn{8}{|l|}{ much work } \\
\hline no & 103 & 103 & 1.00 & reference & 23 & 1.00 & reference \\
\hline yes & 126 & 111 & 0.89 & $0.64-1.23$ & 31 & 1.11 & $0.65-1.92$ \\
\hline \multicolumn{8}{|c|}{ concentration required } \\
\hline no & 70 & 62 & 1.00 & reference & 14 & 1.00 & reference \\
\hline yes & 159 & 152 & 1.08 & $0.76-1.54$ & 40 & 1.25 & $0.70-2.38$ \\
\hline \multicolumn{8}{|l|}{ imposed pace of work } \\
\hline no & 108 & 100 & 1.00 & reference & 23 & 1.00 & reference \\
\hline yes & 121 & 114 & 1.03 & $0.75-1.43$ & 31 & 1.22 & $0.71-2.10$ \\
\hline \multicolumn{8}{|l|}{ uneven pace } \\
\hline no & 131 & 128 & 1.00 & reference & 26 & 1.00 & reference \\
\hline yes & 98 & 86 & 0.90 & $0.64-1.24$ & 28 & 1.43 & $0.84-2.45$ \\
\hline \multicolumn{8}{|l|}{ great physical effort } \\
\hline no & 147 & 138 & 1.00 & reference & 37 & 1.00 & reference \\
\hline yes & 82 & 76 & 1.01 & $0.72-1.42$ & 17 & 0.84 & $0.46-1.47$ \\
\hline \multicolumn{8}{|c|}{ extensive repetition of movements } \\
\hline no & 120 & 117 & 1.00 & reference & 32 & 1.00 & reference \\
\hline yes & 109 & 97 & 0.95 & $0.69-1.32$ & 22 & 0.79 & $0.45-1.35$ \\
\hline \multicolumn{8}{|l|}{ awkward posture } \\
\hline no & 78 & 63 & 1.00 & reference & 21 & 1.00 & reference \\
\hline yes & 151 & 151 & 1.23 & $0.87-1.76$ & 33 & 0.81 & $0.47-1.41$ \\
\hline \multicolumn{8}{|l|}{ walking } \\
\hline no & 223 & 208 & 1.00 & reference & 52 & 1.00 & reference \\
\hline yes & 6 & 6 & 1.13 & $0.39-2.98$ & 2 & 1.54 & $0.25-4.96$ \\
\hline \multicolumn{8}{|l|}{ lengthy sitting posture } \\
\hline no & 82 & 65 & 1.00 & reference & 17 & 1.00 & reference \\
\hline yes & 147 & 149 & 1.26 & 0.89-1.79 & 37 & 1.20 & $0.69-2.18$ \\
\hline \multicolumn{8}{|l|}{ troublesome client } \\
\hline no & 214 & 200 & 1.00 & reference & 47 & 1.00 & reference \\
\hline yes & 15 & 14 & 0.99 & $0.50-1.88$ & 7 & 2.13 & $0.88-4.40$ \\
\hline
\end{tabular}


Table 3. Distribution of persons and sickness absence risk - univariate analyses - cont.

\begin{tabular}{|c|c|c|c|c|c|c|c|}
\hline \multirow[b]{3}{*}{ Variable } & \multirow{3}{*}{$\begin{array}{c}\text { Respondents } \\
(\mathrm{N}=229) \\
{[\mathrm{n}]}\end{array}$} & \multicolumn{6}{|c|}{ Duration of spells } \\
\hline & & \multicolumn{3}{|c|}{ 1-29 days } & \multicolumn{3}{|c|}{$\geq 30$ days } \\
\hline & & $\begin{array}{c}\text { spells } \\
(\mathrm{N}=214) \\
{[\mathrm{n}]}\end{array}$ & $\mathrm{RR}$ & $95 \% \mathrm{CI}$ & $\begin{array}{c}\text { spells } \\
(\mathrm{N}=54) \\
{[\mathrm{n}]}\end{array}$ & $\mathrm{RR}$ & $95 \% \mathrm{CI}$ \\
\hline \multicolumn{8}{|c|}{$\begin{array}{l}\text { Subjective Work Characteristics } \\
\text { Questionnaire Revised (SWCQ-R) }\end{array}$} \\
\hline \multicolumn{8}{|c|}{$\begin{array}{l}\text { Factor } 1 . \text { Tension and pressure } \\
\text { (score) }\end{array}$} \\
\hline low $(1-15)$ & 79 & 70 & 1.00 & reference & 16 & 1.00 & reference \\
\hline average (16-27) & 79 & 64 & 0.89 & $0.59-1.32$ & 16 & 0.96 & $0.48-1.94$ \\
\hline $\operatorname{high}(28-63)$ & 71 & 80 & 1.27 & $0.86-1.88$ & 22 & 1.51 & $0.80-2.93$ \\
\hline \multicolumn{8}{|c|}{ Factor 2. Hazards (score) } \\
\hline low $(1-4)$ & 124 & 99 & 1.00 & reference & 22 & 1.00 & reference \\
\hline average (5-7) & 62 & 76 & 1.49 & $1.03-2.14^{*}$ & 21 & 1.84 & $1.01-3.35^{*}$ \\
\hline high $(8-15)$ & 43 & 39 & 1.12 & $0.72-1.73$ & 11 & 1.42 & $0.66-2.86$ \\
\hline \multicolumn{8}{|c|}{$\begin{array}{l}\text { Factor 3. Intellectual work load } \\
\text { (score) }\end{array}$} \\
\hline low $(1-12)$ & 79 & 75 & 1.00 & reference & 18 & 1.00 & reference \\
\hline average (13-18) & 80 & 70 & 0.87 & $0.59-1.29$ & 15 & 0.77 & $0.38-1.53$ \\
\hline high (19-43) & 70 & 69 & 1.04 & $0.70-1.55$ & 21 & 1.30 & $0.69-2.47$ \\
\hline \multicolumn{8}{|c|}{$\begin{array}{l}\text { Factor 4. Lack of control over work } \\
\text { (score) }\end{array}$} \\
\hline low (1-7) & 55 & 48 & 1.00 & reference & 9 & 1.00 & reference \\
\hline average (8-11) & 80 & 74 & 1.06 & $0.68-1.64$ & 23 & 1.74 & $0.83-3.97$ \\
\hline high (12-28) & 94 & 92 & 1.15 & $0.76-1.76$ & 22 & 1.46 & $0.69-3.34$ \\
\hline \multicolumn{8}{|c|}{$\begin{array}{l}\text { Factor } 5 \text {. Unpleasant working } \\
\text { conditions (score) }\end{array}$} \\
\hline low (1-4) & 109 & 94 & 1.00 & reference & 23 & 1.00 & reference \\
\hline average (5-8) & 71 & 70 & 1.14 & $0.79-1.66$ & 17 & 1.13 & $0.59-2.10$ \\
\hline high (9-21) & 49 & 50 & 1.23 & $0.81-1.86$ & 14 & 1.39 & $0.70-2.67$ \\
\hline \multicolumn{8}{|l|}{ SWCQ-R total (score) } \\
\hline low (1-44) & 73 & 57 & 1.00 & reference & 13 & 1.00 & reference \\
\hline average (45-69) & 85 & 88 & 1.29 & $0.87-1.93$ & 24 & 1.54 & $0.80-3.11$ \\
\hline $\operatorname{high}(70-139)$ & 71 & 69 & 1.25 & $0.82-1.91$ & 17 & 1.34 & $0.65-2.81$ \\
\hline \multicolumn{8}{|c|}{$\begin{array}{l}\text { Lifestyle and non-occupational loads } \\
\text { household chores (h/day) }\end{array}$} \\
\hline$\leq 3$ & 150 & 146 & 1.00 & reference & 44 & 1.00 & reference \\
\hline$\geq 4$ & 79 & 68 & 0.88 & $0.62-1.24$ & 10 & 0.43 & $0.20-0.82 *$ \\
\hline
\end{tabular}


Table 3. Distribution of persons and sickness absence risk - univariate analyses - cont.

\begin{tabular}{|c|c|c|c|c|c|c|c|}
\hline \multirow[b]{3}{*}{ Variable } & \multirow{3}{*}{$\begin{array}{c}\text { Respondents } \\
(\mathrm{N}=229) \\
{[\mathrm{n}]}\end{array}$} & \multicolumn{6}{|c|}{ Duration of spells } \\
\hline & & \multicolumn{3}{|c|}{ 1-29 days } & \multicolumn{3}{|c|}{$\geq 30$ days } \\
\hline & & $\begin{array}{c}\text { spells } \\
(\mathrm{N}=214) \\
{[\mathrm{n}]}\end{array}$ & $\mathrm{RR}$ & $95 \% \mathrm{CI}$ & $\begin{array}{c}\text { spells } \\
(\mathrm{N}=54) \\
{[\mathrm{n}]}\end{array}$ & $\mathrm{RR}$ & $95 \% \mathrm{CI}$ \\
\hline \multirow{2}{*}{\multicolumn{8}{|c|}{$\begin{array}{l}\text { Lifestyle and non-occupational loads - } \\
\text { cont. } \\
\text { shopping [h/day] }\end{array}$}} \\
\hline & & & & & & & \\
\hline$<1$ & 44 & 42 & 1.00 & reference & 8 & 1.00 & reference \\
\hline$\geq 1$ & 185 & 172 & 1.00 & $0.67-1.51$ & 46 & 1.40 & $0.70-3.21$ \\
\hline \multicolumn{8}{|l|}{ active leisure time [h/day] } \\
\hline no leisure time & 122 & 109 & 1.00 & reference & 36 & 1.00 & reference \\
\hline$<1$ & 18 & 8 & 0.53 & $0.23-1.13$ & 5 & 1.01 & $0.35-2.35$ \\
\hline$\geq 1$ & 89 & 97 & 1.23 & $0.88-1.71$ & 13 & 0.50 & $0.26-0.93^{*}$ \\
\hline \multicolumn{8}{|l|}{ age of children at home } \\
\hline \multicolumn{8}{|l|}{$\leq 7$ years } \\
\hline no & 203 & 196 & 1.00 & reference & 48 & 1.00 & reference \\
\hline yes & 26 & 18 & 0.78 & $0.43-1.35$ & 6 & 1.07 & $0.41-2.30$ \\
\hline \multicolumn{8}{|l|}{$8-15$ years } \\
\hline no & 193 & 194 & 1.00 & reference & 44 & 1.00 & reference \\
\hline yes & 36 & 20 & 0.53 & $0.31-0.86$ & 10 & 1.16 & $0.55-2.21$ \\
\hline \multicolumn{8}{|l|}{$\geq 16$ years } \\
\hline no & 187 & 173 & 1.00 & reference & 40 & 1.00 & reference \\
\hline yes & 42 & 41 & 1.00 & $0.66-1.50$ & 14 & 1.46 & $0.77-2.62$ \\
\hline \multicolumn{8}{|c|}{ burden of non-occupational duties } \\
\hline none, low & 88 & 80 & 1.00 & reference & 13 & 1.00 & reference \\
\hline moderate & 108 & 98 & 1.00 & $0.70-1.43$ & 33 & 2.07 & $1.12-4.07^{*}$ \\
\hline high & 33 & 36 & 1.19 & $0.73-1.91$ & 8 & 1.63 & $0.64-3.86$ \\
\hline \multicolumn{8}{|c|}{ opportunity to rest after work } \\
\hline satisfactory & 193 & 178 & 1.00 & reference & 43 & 1.00 & reference \\
\hline lack of opportunities & 36 & 36 & 1.04 & $0.67-1.59$ & 11 & 1.30 & $0.64-2.44$ \\
\hline cigarette smoking & 85 & 75 & 0.93 & $0.66-1.31$ & 15 & 0.67 & $0.36-1.19$ \\
\hline alcohol consumption & 59 & 61 & 1.09 & $0.76-1.56$ & 18 & 1.36 & $0.76-2.37$ \\
\hline \multicolumn{8}{|l|}{ life stress (score) } \\
\hline$\leq 7$ & 101 & 82 & 1.00 & reference & 19 & 1.00 & reference \\
\hline $8-14$ & 56 & 51 & 1.14 & $0.75-1.72$ & 14 & 1.35 & $0.66-2.67$ \\
\hline$\geq 15$ & 72 & 81 & 1.49 & $1.03-2.15^{*}$ & 21 & 1.66 & $0.89-3.11$ \\
\hline
\end{tabular}


Table 3. Distribution of persons and sickness absence risk - univariate analyses - cont.

\begin{tabular}{|c|c|c|c|c|c|c|c|}
\hline \multirow[b]{3}{*}{ Variable } & \multirow{3}{*}{$\begin{array}{c}\text { Respondents } \\
(\mathrm{N}=229) \\
{[\mathrm{n}]}\end{array}$} & \multicolumn{6}{|c|}{ Duration of spells } \\
\hline & & \multicolumn{3}{|c|}{ 1-29 days } & \multicolumn{3}{|c|}{$\geq 30$ days } \\
\hline & & $\begin{array}{c}\text { spells } \\
(\mathrm{N}=214) \\
{[\mathrm{n}]}\end{array}$ & $\mathrm{RR}$ & $95 \% \mathrm{CI}$ & $\begin{array}{c}\text { spells } \\
(\mathrm{N}=54) \\
{[\mathrm{n}]}\end{array}$ & $\mathrm{RR}$ & $95 \% \mathrm{CI}$ \\
\hline \multicolumn{8}{|l|}{ Self-rated health status } \\
\hline \multicolumn{8}{|l|}{ self-rated health } \\
\hline good & 122 & 92 & 1.00 & reference & 17 & 1.00 & reference \\
\hline moderate & 95 & 114 & 1.55 & $1.12-2.15^{*}$ & 33 & 2.42 & $1.37-4.45^{*}$ \\
\hline bad & 12 & 8 & 0.93 & $0.38-2.04$ & 4 & 2.51 & $0.72-6.77$ \\
\hline \multicolumn{8}{|c|}{$\begin{array}{l}\text { Does the health status hamper job } \\
\text { performance? (score) }\end{array}$} \\
\hline yes, very much (1-5) & 154 & 156 & 1.00 & reference & 44 & 1.00 & reference \\
\hline no $(6)$ & 75 & 58 & 0.76 & $0.53-1.08$ & 10 & 0.47 & $0.22-0.89 *$ \\
\hline \multicolumn{8}{|l|}{ disease } \\
\hline no disease & 62 & 43 & 1.00 & reference & 6 & 1.00 & reference \\
\hline disease under treatment & 80 & 84 & 1.54 & $1.00-2.38$ & 26 & 3.38 & $1.49-9.09 *$ \\
\hline disease not treated & 87 & 87 & 1.49 & $0.97-2.29$ & 22 & 2.68 & $1.16-7.92 *$ \\
\hline \multicolumn{8}{|l|}{ occurrence of the diseases ${ }^{\mathrm{a}}$} \\
\hline \multicolumn{8}{|l|}{ accident-related injury } \\
\hline no & 221 & 204 & 1.00 & reference & 50 & 1.00 & reference \\
\hline yes & 8 & 10 & 1.34 & $0.58-2.95$ & 4 & 2.22 & $0.67-5.43$ \\
\hline \multicolumn{8}{|c|}{ musculoskeletal system diseases } \\
\hline no & 146 & 134 & 1.00 & reference & 34 & 1.00 & reference \\
\hline yes & 83 & 80 & 1.07 & $0.76-1.49$ & 20 & 1.05 & $0.60-1.81$ \\
\hline \multicolumn{8}{|l|}{ circulatory system diseases } \\
\hline no & 185 & 165 & 1.00 & reference & 40 & 1.00 & reference \\
\hline yes & 44 & 49 & 1.27 & $0.85-1.88$ & 14 & 1.50 & $0.79-2.69$ \\
\hline \multicolumn{8}{|l|}{ respiratory system diseases } \\
\hline no & 187 & 158 & 1.00 & reference & 39 & 1.00 & reference \\
\hline yes & 42 & 56 & 1.61 & $1.10-2.35^{*}$ & 15 & 1.77 & $0.94-3.14$ \\
\hline \multicolumn{8}{|l|}{ mental disorders } \\
\hline no & 210 & 193 & 1.00 & reference & 48 & 1.00 & reference \\
\hline yes & 19 & 21 & 1.14 & $0.64-1.97$ & 6 & 1.30 & $0.50-2.81$ \\
\hline \multicolumn{8}{|l|}{ nervous system diseases } \\
\hline no & 167 & 160 & 1.00 & reference & 37 & 1.00 & reference \\
\hline yes & 62 & 54 & 0.91 & $0.63-1.32$ & 17 & 1.24 & $0.68-2.17$ \\
\hline
\end{tabular}


Table 3. Distribution of persons and sickness absence risk - univariate analyses - cont.

\begin{tabular}{|c|c|c|c|c|c|c|c|}
\hline \multirow[b]{3}{*}{ Variable } & \multirow{3}{*}{$\begin{array}{c}\text { Respondents } \\
(\mathrm{N}=229) \\
{[\mathrm{n}]}\end{array}$} & \multicolumn{6}{|c|}{ Duration of spells } \\
\hline & & \multicolumn{3}{|c|}{ 1-29 days } & \multicolumn{3}{|c|}{$\geq 30$ days } \\
\hline & & $\begin{array}{c}\text { spells } \\
(\mathrm{N}=214) \\
{[\mathrm{n}]}\end{array}$ & $\mathrm{RR}$ & $95 \% \mathrm{CI}$ & $\begin{array}{c}\text { spells } \\
(\mathrm{N}=54) \\
{[\mathrm{n}]}\end{array}$ & $\mathrm{RR}$ & $95 \% \mathrm{CI}$ \\
\hline \multicolumn{8}{|c|}{$\begin{array}{l}\text { Self-rated health status - cont. } \\
\text { occurrence of the diseases }{ }^{\mathrm{a}} \text { - cont. } \\
\text { digestive system diseases }\end{array}$} \\
\hline no & 189 & 167 & 1.00 & reference & 39 & 1.00 & reference \\
\hline yes & 40 & 47 & 1.31 & $0.87-1.94$ & 15 & 1.77 & $0.95-3.15$ \\
\hline \multicolumn{8}{|c|}{ genitourinary system diseases } \\
\hline no & 202 & 188 & 1.00 & reference & 45 & 1.00 & reference \\
\hline yes & 27 & 26 & 1.00 & $0.60-1.63$ & 9 & 1.45 & $0.66-2.83$ \\
\hline \multicolumn{8}{|l|}{ skin diseases } \\
\hline no & 213 & 200 & 1.00 & reference & 44 & 1.00 & reference \\
\hline yes & 16 & 14 & 0.91 & $0.46-1.71$ & 10 & 2.94 & $1.40-5.59^{*}$ \\
\hline \multicolumn{8}{|l|}{ neoplasms } \\
\hline no & 224 & 206 & 1.00 & reference & 53 & 1.00 & reference \\
\hline yes & 5 & 8 & 1.65 & $0.63-4.19$ & 1 & 0.80 & $0.05-3.64$ \\
\hline \multicolumn{8}{|l|}{ endocrine disorders } \\
\hline no & 212 & 193 & 1.00 & reference & 48 & 1.00 & reference \\
\hline yes & 17 & 21 & 1.34 & $0.75-2.34$ & 6 & 1.55 & $0.59-3.35$ \\
\hline \multicolumn{8}{|c|}{$\begin{array}{l}\text { diseases of blood and blood- } \\
\text { forming organs }\end{array}$} \\
\hline no & 221 & 207 & 1.00 & reference & 51 & 1.00 & reference \\
\hline yes & 8 & 7 & 0.89 & $0.34-2.10$ & 3 & 1.54 & $0.37-4.18$ \\
\hline
\end{tabular}

$\mathrm{RR}$ - rate ratio; $\mathrm{CI}$ - confidence interval.

${ }^{a}$ Occurrence of the diseases was assessed based on an interview; the analysis included those diseases, which were diagnosed before the period of absenteeism.

$* \mathrm{p}<0.05$.

to the married ones (rate ratio $(\mathrm{RR})=1.76,95 \%$ confidence interval (CI): 1.12-2.73). An increased risk of longterm absence ( $\geq 30$ days) was found in the women aged over 40, but it was statistically insignificant.

In the group of workload variables - such as: working time, shift work, overtime work, breaks at work, work with a computer, workstation appropriateness, number of clients served per shift, uncomfortable working position, physical workload - the risk of periods of absence of 1-29 days showed a significant increase among the women employed at "large" post offices (more than 13 customer service workers) and in "small" post offices employing 2-7 workers. This risk was over 2 times higher in the "large" post offices 
employing 13-25 ( $\mathrm{RR}=2.24,95 \% \mathrm{CI}: 1.52-3.33)$ and more than 26 workers ( $\mathrm{RR}=2.33,95 \% \mathrm{CI}$ : $1.43-3.77$ ). In "small" settings, the risk of absence showed an increase of almost $70 \%(\mathrm{RR}=1.69,95 \% \mathrm{CI}: 1.07-2.65)$ compared with the "medium-size" settings (8-12 workers). The risk of absence of 1-29 days was significantly increased $(81 \%)$ in the group of workers employed in a three-shift system compared with a two-shift system $(\mathrm{RR}=1.81,95 \% \mathrm{CI}: 1.24-2.63)$. However, the risk of short-term absence was significantly lower (about 30\%) in the group of part-time workers compared with the fulltime workers ( $\mathrm{RR}=0.66,95 \% \mathrm{CI}$ : $0.46-0.94$ ).

The risk of long-term absence increased along with the increasing time of work with a computer; if working time exceeded $6.5 \mathrm{~h}$, the risk was over 2.5 times higher compared to the working time of less than $4 \mathrm{~h}$ $(\mathrm{RR}=2.57,95 \% \mathrm{CI}:$ 0.92-10.75). This risk was over 3.5 times higher when the number of customers per shift exceeded 50 people: for the interval of 51100 people the risk was $\mathrm{RR}=3.68,95 \%$ CI: $1.11-$ 22.81, and it was $77 \%$ higher in a three-shift system $(\mathrm{RR}=1.77,95 \% \mathrm{CI}: 0.96-3.13)$. The risk of long-term absence was over $60 \%$ lower in the group of women who spent less than $25 \%$ of their working time in a slightly bent body position compared with the women who worked in this position for over $75 \%$ of their working time ( $\mathrm{RR}=0.37,95 \% \mathrm{CI}: 0.13-0.88)$.

In the group of variables of subjective rates of workload, such as fatigue at work, negative factors at work (e.g., job performance at different times of the day, commuting to work, large amount of work, the need to concentrate, imposed pace of work, uneven pace of work, great physical effort, extensive repetition of movements, forced work position, walking around at work, longtime sitting position, troublesome customers), a significantly enhanced rate of absence spells of 1-29 days ( $\mathrm{RR}=1.48,95 \% \mathrm{CI}$ : 1.02 2.13) was observed in the case of "work at different times of the day" factor. A lengthy sitting posture and an awkward posture also increased, albeit insignificantly, the rate of absence spells ( $\mathrm{RR}=1.26,95 \% \mathrm{CI}: 0.89-1.79$ and $\mathrm{RR}=1.23,95 \% \mathrm{CI}: 0.87-1.76$, respectively).

Extreme fatigue contributed to a 2-fold increase in the rate of long-term absence spells of 30 days and more $(\mathrm{RR}=2.06$, 95\% CI: 0.96-5.1); however, these values were statistically insignificant. The same applied to another tiring factor at work, i.e., "troublesome customers" ( $\mathrm{RR}=2.13,95 \%$ CI: 0.88-4.4).

In view of the results obtained, it should be acknowledged that, basically, occupational stress did not increase the risk of absence spells. A statistically significant increase in the risk of absence was only observed in the case of "hazards at work." However, the results are contradictory. An enhanced risk of both kinds of absence spells (longand short-term) was observed at a moderate level of stress resulting from the presence of hazards at work, such as: exposure to harmful factors or the risk of an accident, shift work, excessive noise and inappropriate temperature (a $49 \%$ increase in the risk of short-term absence and an $84 \%$ increase in long-term absence), whereas a high level of stress induced by this factor did not significantly increase the risk.

In the group of variables which characterize non-occupational burdens (the number of hours devoted to household chores, shopping, or active leisure time, the number of children, including their age, subjectively assessed burden of non-occupational duties, assessment of an opportunity to rest after work) and lifestyle-related variables (cigarette smoking, alcohol consumption) there were factors that significantly influenced the risk of long-term absence spells. Active leisure time of over one hour per day contributed to a $50 \%$ decrease $(\mathrm{RR}=0.5,95 \% \mathrm{CI}: 0.26-0.93)$ and performance of household chores for over $4 \mathrm{~h}$ per day a reduction of almost $60 \%$ in the long-term ( $\geq 30$ days) absence risk $(\mathrm{RR}=0.43,95 \% \mathrm{CI}: 0.2-0.82)$. The burden of non-occupational duties, assessed by female workers as moderate, 
caused a 2-fold increase in the risk of long-term absence $(\mathrm{RR}=2.07,95 \% \mathrm{CI}: 1.12-4.07)$.

The analyses of life stress measured by the intensity of negative emotions associated with the occurrence of stressful life events showed that a high level contributes to an increase by almost $50 \%$ in the risk of short-term absence spells ( $\mathrm{RR}=1.49,95 \% \mathrm{CI}$ : 1.03-2.15); however, life stress was not found to be a factor affecting long-term absence spells.

In the group of variables which characterize health status, namely the occurrence of diseases and complaints prior to the absence spells reported by the respondents, the occurrence of respiratory diseases significantly increased the risk of absence spells of 1-29 days $(\mathrm{RR}=1.61,95 \% \mathrm{CI}: 1.1-2.35)$, whereas the occurrence of skin diseases significantly increased the long-term absence (RR $=2.94,95 \%$ CI: 1.4-5.59).

Self-rated health status, i.e., the variable used by the women to define their health status as moderate was significantly related to an increased risk of short- and longterm absence spells ( $\mathrm{RR}=1.55,95 \% \mathrm{CI}: 1.12-2.15$ and $\mathrm{RR}=2.42,95 \%$ CI: 1.37-4.45, respectively) compared to self-rated good health. Self-rated health and its effect on job performance was a statistically significant factor that affected the rate of long-term absence spells; if job performance was not hampered by health status, the absence rate was $53 \%$ lower compared with job performance greatly hampered by the health condition ( $\mathrm{RR}=0.47,95 \%$ CI: 0.22-0.89).

Occurrence of any disease significantly increased the risk of long-term absence compared with the absence of a disease, regardless of whether or not a given disease was treated ( $\mathrm{RR}=3.38,95 \%$ CI: $1.49-9.09$ and $\mathrm{RR}=2.68,95 \% \mathrm{CI}: 1.16-7.92$, respectively). A similar relationship was also observed for short-term absence spells; however, the absence risk was lower and statistically significant when the disease was treated ( $\mathrm{RR}=1.54,95 \%$ CI: $1-2.38)$.

\section{Multivariable analysis}

A multivariable model of the risk of absence spells of 1-29 days is presented in Table 4. In this model the following variables had a statistically significant impact on the increased risk of absence:

- marital status - in the case of the unmarried women the risk was over twice as high as in the case of the married ones $(\mathrm{RR}=1.56,95 \% \mathrm{CI}$ : 1.01-2.39);

- the number of employees in a given post office in post offices employing 13-25 customer service workers, the risk of absence spells was over 2 times higher than in those employing 8-12 workers $(\mathrm{RR}=2.03,95 \% \mathrm{CI}: 1.41-2.93)$. In those employing over 26 workers, the risk was almost 2 times higher $(\mathrm{RR}=1.82,95 \%$ CI: 1.15-2.88), whereas in those employing fewer than 8 workers a $60 \%$ increase in the risk was noted ( $\mathrm{RR}=1.6,95 \%$ CI: 1.04-2.42);

- work at different times of the day, considered by female workers as a tiring factor, caused an increase of over $50 \%$ in the absence risk ( $\mathrm{RR}=1.57,95 \% \mathrm{CI}: 1.14-2.14)$;

- breaks depending on current needs were associated with a $50 \%$ increase in the absence risk, compared with regular breaks $(\mathrm{RR}=1.5,95 \% \mathrm{CI}$ : 1.07-2.07);

- self-rated health status - health assessed as moderate was associated with a $71 \%$ increase in the absence risk $(\mathrm{RR}=1.71,95 \% \mathrm{CI}: 1.25-2.32)$ compared with good health;

- occurrence of respiratory diseases was associated with a 50\% increase in the absence risk (RR = 1.51, 95\% CI: 1.08-2.08);

- SWCQ-R factors - hazards at work, occupational stress resulting from the presence of health hazards at work did not significantly increase the absence risk; in the context of other variables this predictor lost its importance;

- similarly, life stress did not prove to be an important predictor of absence spells in the multivariable model of the short-term absence risk. 
Table 4. Multivariable model of sickness absence risk for spells of 1-29 days among the female post office clerks

\begin{tabular}{|c|c|c|}
\hline \multirow{2}{*}{ Variable } & \multicolumn{2}{|c|}{ Spells of 1-29 days } \\
\hline & $\mathrm{RR}$ & $95 \% \mathrm{CI}$ \\
\hline \multicolumn{3}{|l|}{ Marital status } \\
\hline married & 1.00 & reference \\
\hline unmarried & 1.56 & $1.01-2.39 *$ \\
\hline cohabiting & 1.47 & $0.80-2.60$ \\
\hline divorced, widowed & 1.03 & $0.67-1.55$ \\
\hline \multicolumn{3}{|l|}{ Post office clerks (female) [n] } \\
\hline$\leq 7$ & 1.60 & $1.04-2.42 *$ \\
\hline $8-12$ & 1.00 & reference \\
\hline $13-25$ & 2.03 & $1.41-2.93^{*}$ \\
\hline$\geq 26$ & 1.82 & $1.15-2.88^{*}$ \\
\hline \multirow{2}{*}{\multicolumn{3}{|c|}{$\begin{array}{l}\text { Self-assessment of tiring factors at work } \\
\text { work at different times of the day }\end{array}$}} \\
\hline & & \\
\hline no & 1.00 & reference \\
\hline yes & 1.57 & $1.14-2.14 *$ \\
\hline \multicolumn{3}{|l|}{ Breaks at work } \\
\hline set in working time regulation & 1.00 & reference \\
\hline free choice, depending on the needs & 1.50 & $1.07-2.07^{*}$ \\
\hline no breaks & 1.10 & $0.68-1.72$ \\
\hline \multicolumn{3}{|l|}{ SWCQ-R, Factor 2. Hazards (score) } \\
\hline low $(1-4)$ & 1.00 & reference \\
\hline average (5-7) & 1.28 & $0.93-1.74$ \\
\hline $\operatorname{high}(8-15)$ & 0.71 & $0.47-1.05$ \\
\hline \multicolumn{3}{|l|}{ Self-rated health } \\
\hline good & 1.00 & reference \\
\hline moderate & 1.71 & $1.26-2.32^{*}$ \\
\hline bad & 1.14 & $0.50-2.31$ \\
\hline \multicolumn{3}{|c|}{ Occurrence of the respiratory system diseases } \\
\hline no & 1.00 & reference \\
\hline yes & 1.51 & $1.08-2.08 *$ \\
\hline
\end{tabular}

$\mathrm{RR}$ - rate ratio; CI - confidence interval.

$* \mathrm{p}<0.05$.

Table 5 presents a model of long-term absence spells ( $\geq 30$ days). In this model the variable defined as the number of customers per day exerts the highest statistically significant impact on the increase in the longterm absence risk. If the number of customers in this group ranged from 51-150, the risk increased by over 3 times 
Table 5. Multivariable model of sickness absence risk for spells of $\geq 30$ days among the female post office clerks

\begin{tabular}{lll}
\hline \multirow{2}{*}{ Variable } & \multicolumn{2}{c}{ Spells of $\geq 30$ days } \\
\cline { 2 - 3 } Shift work [n of shifts] & RR & 95\% CI \\
1 & & \\
2 & 0.35 & $0.02-1.66$ \\
3 & 1.00 & reference \\
Clients [n/shift] & 1.82 & $0.97-3.29$ \\
$\leq 50$ & & \\
$51-100$ & 1.00 & reference \\
$101-150$ & 3.62 & $1.07-22.58^{*}$ \\
$\geq 151$ & 3.26 & $0.92-20.68$ \\
Performance of household chores [h/day] & 1.68 & $0.36-11.82$ \\
$\quad \leq 3$ & & \\
$\geq 4$ & 1.00 & reference \\
Self-rated health & 0.40 & $0.18-0.79^{*}$ \\
good & & \\
moderate & 1.00 & reference \\
bad & 1.97 & $1.06-3.78^{*}$ \\
Occurrence of the skin diseases & 2.33 & $0.66-6.42$ \\
no & & \\
yes & 1.00 & reference \\
\hline
\end{tabular}

$\mathrm{RR}$ - rate ratio; $\mathrm{CI}$ - confidence interval. $* \mathrm{p}<0.05$.

compared with the lower number of customers served by one worker ( $\mathrm{RR}=3.62,95 \% \mathrm{CI}$ : 1.07-22.58). Moreover, past skin diseases resulted in over 2-fold increase in the absence risk ( $\mathrm{RR}=2.02,95 \%$ CI: 0.91-4.13). Selfrated health status also significantly influenced occurrence of long-term absence - the worse the health rating, the higher the risk; for moderate self-rated health the risk increased about 2-fold (RR $=1.97,95 \%$ CI: 1.06-3.78).

Time spent on household chores proved to be a variable that significantly decreased the occurrence of long-term absence spells; a $60 \%$ decrease in the absence risk was noted if a woman devoted more than $4 \mathrm{~h}$ a day to these duties ( $\mathrm{RR}=0.4,95 \% \mathrm{CI}: 0.18-0.79)$.

\section{DISCUSSION}

\section{Comparison with other studies}

Final models obtained from the analyses identify sickness absence predictors in the women employed in postal customer service in a large urban agglomeration. They concern a large number of factors that may affect sickness absence risk, and are discussed in reports from relevant studies carried out in other countries.

Among the analyzed demographic variables a significantly higher risk of absence spells of 1-29 days was observed among the unmarried women compared with the married ones. However, findings of other studies on the relationship between marital status of women and their sickness 
absence are contradictory. For example, a Japanese study has revealed that the risk of absence among unmarried, divorced and widowed women was higher than among married women [27]. On the other hand, studies carried out on the female population of Malaysia have revealed a significantly increased absence risk among married women [28]. Studies in the French GAZEL (GAZ and ELectricité) cohort have shown the highest average annual number of sickness absence days among divorced and separated women and widows, while absence spells among wives and unmarried women were at a similar, lower level [29].

The risk of absence spells of 1-29 days was significantly associated with the size of a given post office, namely the number of postal workers employed. The bigger the setting, the higher the risk of absence spells. This observation has been reflected in the studies of Italian [30] and Swedish postal workers [17,18].

In the group of variables concerning characteristics of physical working conditions, work at different times of the day, regarded by women as a tiring factor at work, had significant impact on the risk of absence of 1-29 days. This variable was closely related to shift work that had a significant influence on the increase in the risk of long-term absence ( $\geq 30$ days).

The results of Norwegian studies carried out on a group of workers employed in the chemical industry have not revealed any effect of shift work on absence caused by the major categories of diseases [31], while an Italian study has shown a $40 \%$ decrease in the risk of absence of 7 days and longer among women working shifts (regardless of the number of shifts) compared with those working a single shift (no shifts) [30].

The effect of working conditions expressed as a variable "breaks at work" in the model of short-term absence (1-29 days), in which a 50\% increase in the risk in the case of "breaks at work according to workers' needs," which may have indirectly reflected the workload; as well as workload expressed as the number of customers served during one shift indicated in the model of long-term absence risk, has not been indicated by other studies reported in the literature. However, physical working conditions, such as: heavy physical work, lifting heavy objects, twisting the neck and back, monotonous movements of the body, manual work and an uncomfortable position at work are the subject of numerous analyses, which indicate their effect on sickness absence in many occupational groups, e.g., Swedish postal workers [17,18], Italian or Danish workers [30,32,33].

In our study, which was carried out in the group of women working in postal customer service, in spite of taking into account many such variables (e.g., uncomfortable position at work, workload, overtime work) in the baseline analysis, in the final model they did not prove to be significant predictors of sickness absence.

Results of our study related to the relationship between stress and sick leave are not consonant with the findings of other researchers. For example Moreau et al. [34] have found that job stress and low social support at work were related to repetitive spells of sickness in both sexes, with odds ratios of 1.32 (99\% CI: 1.04-1.68) in men and 1.61 (99\% CI: 1.13-2.33) in women. Similar patterns of results have been obtained in the study of Swedish women [35].

Also in the literature review on determinants of sicknessabsence performed by Beemsterboer et al. [36], both life and occupational stress have been identified as factors affecting sick-leave frequency and duration. The reason for these contradictory findings may be related to the strategy of data analysis. The relationship between stress and sickness absence is stable when it is analyzed separately from other factors related to work and private life [37]. In the multivariable analyses the perceived stress effect may be significantly reduced, as observed in our study.

In the group of variables, which characterize lifestyle and non-occupational burdens, only one variable, namely performance of household chores for $4 \mathrm{~h}$ a day, significantly reduced the risk of long-term absence spells. This may 
be related to the so called occupational selection, which means that this group only comprised women in good health, and those able to cope with their household chores and occupational work without conflict. It should be emphasized here, that in French studies a high level of work and family demands, measured by occupational burdens and the number of family dependants, significantly increased the risk of sickness absence [29].

Another variable, i.e., active leisure time reduced the risk of long-term absence spells, as in the Danish study [38]. However, it was not confirmed in the multivariable model. Neither lifestyle characteristics, such as cigarette smoking and alcohol consumption, nor the number of children in the family, including their age [39] confirmed their effect on the risk of absence in the group under the study. This result is in contradiction with other studies [18,29,40,41]. The group of variables, which characterize health status significantly determined the frequency of both long- and short-term (1-30 days) absence spells. In the multivariable model, these are the self-rating of health status and the occurrence of some chronic diseases that almost double the risk of sickness absence. This relationship has been confirmed by numerous studies [20,27,32]. For example, a Danish study has revealed that the low self-rated health status caused a $69 \%$ increase in the absence risk [32]; and the study of Dutch university workers has shown that subjective complaints considerably increased the risk of absence both in workers with chronic diseases and those with other illnesses [20].

The results presented for this research showed that in the group of female postal workers directly involved in customer service (postal assistants and clerks), working conditions and self-rated health status proved to be significant predictors of sickness absence, which is in accordance with numerous studies reported in the literature. It is also worth referring to the Helsinki study, in which physical domains of functioning, in particular the ability to perform daily activities, pain and general health, were the major predictors of sickness absence of over 2 weeks among workers aged 40-60 [42].

\section{Limitations}

Some limitations of the study may explain the rather small group of statistically significant variables, which were selected in the final models. Other factors that may influence the selected predictors for the multivariable models are the applied methods of models construction concerning model selection criteria (Akaike information criterion - AIC, Bayesian information criterion - BIC) as well as the way of including the variables into the model (forward stepwise, penalized regression). Therefore, to evaluate sensitivity of the obtained results, also other methods for multivariable models construction, such as stepwise method with BIC criterion [43] and Elastic Net regression, were used [44]. Those methods were not restricted to the predictors selected by the univariate analyses. Comparison of the obtained results showed a considerable compliance of the selected significant predictors in the analyzed models of long- and short-term sickness absence.

In fact, the study was carried out during a period of planned postal service restructuring in Poland, which had a significant impact on the questionnaire-based survey; $10 \%$ of the study subjects refused to respond due to the fear of losing their jobs. This may have led to some bias. This is, to a certain extent, confirmed by the level of sickness absence defined for the whole random sample, which was slightly higher as compared to the absence level in the group interviewed.

Causes of sickness absence not mentioned in the medical absence certificates issued for the employer were another limitation of the study (in Poland each case of absence has to be documented). Therefore, causes of diseases were established on the basis of the questionnaire-based interview. Despite these limitations, the results can play a significant role in structural and organizational changes in post offices [45]. 
An interesting, as well as a controversial finding related to breaks at work, observed in this study, may serve here as an example. Let us remember that the risk of short-term absence (1-29 days) increased when workers could freely decide about breaks at work. In the context of the available data [46,47], the freedom of taking decision, when break at work is one of its aspects, should be positively associated with health and in consequence, be translated into a lower risk of absence. However, in our studies, strict regime concerning breaks at work (no freedom of taking decisions) was associated with a lower risk of short-term absence (1-29 days). It is likely that setting restrictive rules on breaks at work by superiors is a sign of a dominant authoritative style of administration, primarily oriented towards productivity.

In such conditions workers can be convinced that making use of medical absence certificates is perceived by a superior as something negative and decide to attend work while being sick. In the literature, this phenomenon is known as presenteeism; one of its determinants is putting pressure on productivity and the attitude of managers towards sickness absence management in the company [48-50].

\section{CONCLUSIONS}

Absence indicator, expressed in days, increased after 30 years of age, whereas the frequency of absence was the highest in the youngest and in the oldest age groups. The highest indicators were noted in the group of women with the shortest duration of employment.

The risk of short-term sickness absence was also higher, in the case of higher workload (work in a small unit - the necessity to do many various tasks, and work in a large unit the number of clients that have to be served) and/or an unfavorable work organization (work in a 3-shift system), as well as the incidence of respiratory system diseases and not very good self-rated state of health. The risk of long-term sickness absence was increased by the number of clients $>50$ up to 150 of them, as well as by a deteriorating subjective assessment of the state of health and the incidence of skin diseases. The reduced risk of such absence occurred in the case of individuals who can still devote time to household chores for more than $4 \mathrm{~h}$ a day. The present study demonstrated that sickness absence, apart from the obvious relationship with evaluation of health status and occurrence of certain diseases, is also associated with the workload and working in a 3-shift system. This suggests that rationalization of working conditions is an appropriate action towards improvement of the health of workers and reduction of absenteeism.

\section{REFERENCES}

1. Encyclopaedia of occupational health and safety. Vol. 1. Geneva: International Labour Office; 1983. p. 5-10.

2. Whitaker SC. The management of sickness absence. Occup Environ Med. 2001;58:420-4, http://dx.doi.org/10.1136/ oem.58.6.420.

3. Prins R, de Graaf A. Comparison of sickness absence in Belgian, German and Dutch firms. Br J Ind Med. 1986;43:529_ 36, http://dx.doi.org/10.1136/oem.43.8.529.

4. Kivimäki M, Vahtera J, Thomson L, Griffiths A, Cox T, Pentti J. Psychosocial factors predicting employee sickness absence during economic decline. J Appl Psych. 1997;82(6):858-72, http://dx.doi.org/10.1037/0021-9010.82.6.858.

5. Johns G. Presenteeism in the workplace: A review and research agenda. J Organ Behav. 2010;31:519-42, http://dx.doi. org/10.1002/job.630.

6. Gustafsson K, Marklund S. Consequences of sickness presence and sickness absence on health and work ability: A Swedish prospective cohort study. Int J Occup Med Environ Health. 2011;24(2):153-65, http://dx.doi.org/10.2478/ s13382-011-0013-3.

7. Searle SJ. Sickness absence and duration of service in Post Office 1982-3. Br J Ind Med. 1986;43:458-64, http://dx.doi. org/10.1136/oem.43.7.458.

8. Alexanderson K, Leijon M, Akerlind I, Rydh H, Bjurulf P. Epidemiology of sickness absence in a Swedish county in 1985, 1986, and 1987. Scand J Soc Med. 1994;22:27-34. 
9. Ihlebaek C, Brage S, Eriksen HR. Health complaints and sickness absence in Norway, 1996-2003. Occup Med. 2007;57:43-9, http://dx.doi.org/10.1093/occmed/kql107.

10. European Foundation for the Improvement of Living and Working Conditions. Preventing absenteeism at the workplace. Research summary. Luxembourg: Office for Official Publications of the European Communities; 1997.

11. Lidwall U, Marklund S. Trends in long-term sickness absence in Sweden 1992-2008: The role of economic conditions, legislation, demography, work environment and alcohol consumption. Int J Soc Welfare. 2011;20:167-79, http:// dx.doi.org/10.1111/j.1468-2397.2010.00744.x.

12. Johansen K, Bihrmann K, Mikkelsen S, Lynge E. Trends in sickness absence in Denmark. Scand J Work Environ Health. 2009;35(5):334-41, http://dx.doi.org/10.5271/ sjweh.1346.

13. Houdmont J, Kerr R, Addley K. Psychosocial factors and economic recession: The Stormont Study. Occup Med. 2012;62:98-104, http://dx.doi.org/10.1093/occmed/kqr216.

14. Cahili J, Landsbergis PA. Job strain among post office mailhandlers. Int J Health Serv. 1996;26(4):731-50.

15. Jacobson BH, Aldana SG, Goetzel Vardell KD, Adams TB, Pietras RJ. The relationship between perceived stress and self-reported illness-related absenteeism. Am J Health Promot. 1996;11(1):54-61, http://dx.doi.org/10.4278/0890-117111.1.54.

16. Szubert Z, Szeszenia-Dąbrowska N, Sobala W. [The effect of the system transformations on sick absenteeism in the workplace. II. Causes of work disability among persons under termination of employment]. Med Pr. 1998;6:517-25. Polish.

17. Voss M, Floderus B, Diderischen F. Physical, psychosocial, and organisational factors relative to sickness absence: A study based on Sweden Post. Occup Environ Med. 2001; 58:178-84, http://dx.doi.org/10.1136/oem.58.3.178.

18. Voss M, Floderus B, Diderischen F. How do job characteristics, family situation, domestic work, and lifestyle factors relate to sickness absence? A study based on Sweden
Post. J Occup Environ Med. 2004;46:1134-43, http://dx.doi. org/10.1097/01.jom.0000145433.65697.8d.

19. Ala-Mursula L, Vahtera J, Pentti J, Kivimäki M. Effect of employee worktime control on health: A prospective cohort study. Occup Environ Med. 2004;61:254-61, http:/dx.doi. org/10.1136/oem.2002.005983.

20. Roskes K, Donders NCGM, van der Gulden JWJ. Healthrelated and work-related aspects associated with sick leave: A comparison of chronically ill and non-chronically ill workers. Int Arch Occup Environ Health. 2005;78:270-8, http:// dx.doi.org/10.1007/s00420-004-0596-0.

21. Dudek B, Waszkowska M, Merecz D, Hanke W. [Protection of workers against occupational stress]. Łódź: Instytut Medycyny Pracy; 2004. Polish.

22. Holmes TH, Rahe RH. Social readjustmet rating scale. J Psychosom Res. 1967;11:213-8.

23. Good PI. Resampling methods: A practical guide to data analysis. Boston: Birkhäuser; 1999, http://dx.doi. org/10.1007/978-1-4757-3049-4.

24. Akaike H. Information theory and an extension of maximum likelihood principle. In: Petrov BN, Csaki F, editors. Information theory: Proceedings of the Second International Symposium. Budapest: Akademiai Kiado; 1973. p. 267-81.

25. R Development Core Team. R: A language and environment for statistical computing. Vienna: R Foundation for Statistical Computing; 2011.

26. Venables WN, Ripley BD. Modern applied statistics with S. 4th ed. New York: Springer; 2002, http://dx.doi. org/10.1007/978-0-387-21706-2.

27. Ishizaki M, Kawakami N, Honda R, Nakagawa H, Morikawa Y, Yamada Y. Psychosocial work characteristics and sickness absence in Japanese employees. Int Arch Occup Environ Health. 2006;79:640-6, http://dx.doi.org/10.1007/ s00420-006-0095-6.

28. Chee HL, Rampal KG. Relation between sick leave and selected exposure variables among women semiconductor workers in Malaysia. Occup Environ Med. 2003;60:262-70, http://dx.doi.org/10.1136/oem.60.4.262. 
29. Sabbath EL, Melchior M, Goldberg M, Zins M, Berkman LF. Work and family demands: Predictors of all-cause sickness absence in the GAZEL cohort. Eur J Public Health. 2012;22(1):101-6, http://dx.doi.org/10.1093/eurpub/ ckr041.

30. D'Errico A, Costa G. Socio-demographic and work-related risk factors for medium- and long-term sickness absence among Italian workers. Eur J Public Health. 2012;22(5): 683-8, http://dx.doi.org/10.1093/eurpub/ckr140.

31. Kleiven M, Boggild H, Jeppesen HJ. Shift work and sick leave. Scand J Work Environ Health. 1998;24 Suppl. 3:128-33.

32. Labriola M, Lund T, Burr H. Prospective study of physical and psychosocial risk factors for sickness absence. Occup Med. 2006;56:469-74.

33. Lund T, Labriola M, Christensen KB, Bültmann U, Villadsen E. Physical work environment risk factors for long term sickness absence: Prospective findings among a cohort of 5357 employees in Denmark. BMJ. 2006;332:449-52, http://dx.doi.org/10.1136/bmj.38731.622975.3A.

34. Moreau M, Valente F, Mak R, Pelfrene E, de Smet P, de Backer $\mathrm{G}$, et al. Occupational stress and incidence of sick leave in the Belgian workforce: The Belstress study. J Epidemiol Community Health. 2004;58:507-16, http://dx.doi. org/10.1136/jech.2003.007518.

35. Holmgren K, Dahlin-Ivanoff S, Björkelund C, Hensing G. The prevalence of work-related stress, and its association with self-perceived health and sick-leave, in a population of employed Swedish women. BMC Public Health. 2009;9: 73-83, http://dx.doi.org/10.1186/1471-2458-9-73.

36. Beemsterboer W, Stewart R, Groothoff J, Nijhuis F. A literature review on sick leave determinants (1984-2004). Int J Occup Med Environ Health. 2009;22(2):169-79, http:// dx.doi.org/10.2478/v10001-009-0013-8.

37. Szubert Z, Merecz-Kot D, Sobala W. [Occupational stress and the risk of sickness absence in customer service workers]. Med Pr. 2009;60(4):259-71. Polish.

38. Bernaards CM, Jans MP, van den Heuvel SG, Hendriksen IJ, Houtman IL, Bongers PM. Can strenuous leisure time physical activity prevent psychological complaints in a working population? Occup Environ Med. 2006;63:10-6, http:// dx.doi.org/10.1136/oem.2004.017541.

39. Floderus B, Hagman M, Aronsson G, Marklund S, Wikman A. Medically certified sickness absence with insurance benefits in women with and without children. Eur J Public Health. 2012;22(1):85-92, http://dx.doi.org/10.1093/eurpub/ckr028.

40. Eriksen W, Natving B, Rutle O, Bruusgaard D. Smoking as a predictor of long-term work disability in physically active and inactive people. Occup Med. 1998;5:315-20, http:// dx.doi.org/10.1093/occmed/48.5.315.

41. Laaksonen M, Piha K, Martikainen P, Rahkonen O, Lahelma E. Health-related behaviours and sickness absence from work. Occup Environ Med. 2009;66:840-7, http://dx.doi. org/10.1136/oem.2008.039248.

42. Laaksonen M, Kääriä SM, Leino-Arjas P, Lahelma E. Different domains of health functioning as predictors of sickness absence - A prospective cohort study. Scand J Work Environ Health. 2011;37(3):213-8, http://dx.doi.org/10.5271/ sjweh.3131.

43. Schwarz G. Estimating the dimension of a model. Ann Stat. 1978;6(2):461-4, http://dx.doi.org/10.1214/aos/1176 344136.

44. Zou H, Hastie T. Regularization and variable selection via the elastic net. J R Stat Soc B. 2005;67(2):301-20, http:// dx.doi.org/10.1111/j.1467-9868.2005.00503.x.

45. Wahlstedt KG, Nygård CH, Kemmlert K, Torgén M, Björkstén MG. The effects of a change in work organization upon the work environment and musculoskeletal symptoms among letter carriers. Int J Occup Saf Ergon. 2000;6(2): 237-55, http://dx.doi.org/10.1080/10803548.2000.11076454.

46. Karasek RA, Theorell T. Healthy work: Stress, productivity and the reconstruction of working life. New York: Basic Books; 1990.

47. Theorell T, Karasek RA. Current issues relating to psychosocial job strain and cardio-vascular disease research. J Occup Health Psychol. 1996;1:9-26, http://dx.doi.org/10.1037/10768998.1.1.9. 
48. Baker-McClearn D, Greasley K, Dale J, Griffith F. Absence management and presenteeism: The pressures on employees to attend work and the impact of attendance on performance. Hum Resour Manag J. 2010;20(3):311-28, http:// dx.doi.org/10.1111/j.1748-8583.2009.00118.x.

49. Aronsson, G, Gustafsson K. Sickness presenteeism: Prevalence, attendance-pressure factors and an outline of a model for research. J Occup Environ Med. 2005;47(9):958-66, http://dx.doi.org/10.1097/01.jom.0000177219.75677.17.

50. Gilbreath B, Karimi L. Supervisor behavior and employee presenteeism. Int J Leadersh Stud. 2012;7(1):114-31.

This work is available in Open Access model and licensed under a Creative Commons Attribution-NonCommercial 3.0 Poland License - http://creativecommons.org/ licenses/by-nc/3.0/pl/deed.en. 